Pacific Journal of Mathematic 


\title{
THE UNLOADING PROBLEM \\ FOR SEVERELY TWISTED BARS
}

\section{TSUAN Wu TING}

\begin{abstract}
Suppose that a cylindrical bar is twisted by terminal couples beyond its elastic range. What will happen if the applied load is (gradually) removed? This question will be formulated, according to the deformation theory of plasticity, as a problem of variational inequalities over a convex set in a Hilbert space and the existence, uniqueness and the regularity of the solution will be established. In addition, physically relevant results will be derived.
\end{abstract}

1. Introduction. If the bar is twisted in the elastic range only, then the behavior is well-known. For elastic-plastic torsion, i.e., torsion beyond the elastic range of the material, the problem was also essentially solved by various authors during the last decade $[1,2,4,9,10,13,15]$. However, these results are applicable only when the applied torque is a continuous nondecreasing function of time. This case is known as the "loading process". The question we raise here is about the "unloading process". This question has an immediate answer if the applied torque is sufficiently small or if the material is perfectly elastic. However, the answer is not obvious if the material is elastic-plastic and if the applied torque is so large as to cause permanent "set".

Throughout this paper, .we shall denote by $G$ a plane domain describing the cross-section of the bar. We state our restrictions on $G$ explicitly as follows:

(i) $\partial G$, the boundary of $G$, is a twice continuously differentiable Jordan curve;

(ii) The curvature of $\partial G$, as a function of its arc length, assumes only a finite number of maxima and minima;

(iii) $G$ is simply connected.

We present here three equivalent formulations of the unloading process. The basic existence and uniqueness questions are settled in $\S 4$ and the equivalence of the different formulations are proved in $\$ \S 6,9$. To eliminate the inequality constraints, two types of Lagrange's multipliers are introduced. Their existence and uniqueness are established in $\S \S 5,8$, 9 and 12. Since the problem is formulated as a problem for determining the stresses in what is known as the dual problem, we are obliged to consider the corresponding primary problem for determining the displacement field. This is carried out in $\$ 12$ by first solving the topological elastic-plastic partition problem in $\$ 11$. 
2. Known facts on the loading problem. We state the loading problem together with known results. Let $C_{0}^{\infty}(G)$ be the class of infinitely smooth functions with compact support in $G$. For $f$ and $g$ in $G$, we write

$$
\begin{aligned}
& (f, g)_{0} \equiv \int_{G} f(x) g(x) d x, \\
& (f, g)_{1} \equiv(f, g)_{0}+\int_{G} \operatorname{grad} f(x) \cdot \operatorname{grad} g(x) d x,
\end{aligned}
$$

where $x \equiv\left(x_{1}, x_{2}\right)$ and $d x \equiv d x_{1} d x_{2}$. The corresponding $L^{2}$-norm and the Dirichlet norm is denoted by $\|\cdot\|_{\alpha}, \alpha=0,1$. As usual, the completion of $C_{0}^{\infty}(G)$ under $\|\cdot\|_{1}$-norm - is denoted by $H_{0}^{1}(G)$ which is a separable Hilbert space.

Let $k$ be a positive (yield) constant and let

$$
Y=\left\{u ; u \in H_{0}^{1}(G),|\operatorname{grad} u| \leqq k \text { a.e. in } G\right\} .
$$

Then $Y$ is a closed convex subset of $H_{0}^{1}(G)$ [4]. The loading problem is to find a function $\psi^{*}$ in $Y$ such that

$$
I\left[\psi^{*}\right]=\inf _{Y} I[u]=\inf _{Y} \int_{G}\left[|\operatorname{grad} u|^{2}-4 \mu \theta u\right] d x,
$$

where $\mu$ and $\theta$ positive constants standing for the shear modulus and the angle of twist per unit length, respectively.

The minimizer $\psi^{*}$ is known as the Prandtl stress function and $\operatorname{grad} \psi^{*}$ represents the nonzero components of the stresses. The yielding constant $k$ in (2.2) is the upper bound for the maximum shearing stress and is also the upper bound of distortion energy density.

Let $C^{k+\alpha}(G)$ be the class of functions whose derivatives of order $\leqq k$ are Hölder continuous in $G$ with exponent $\alpha, 0<\alpha<1$. Then we have the following existence, uniqueness and regularity results $[1,2,9,15]$.

THEOREM 2.1. The loading problem (2.3) has a unique minimizer $\psi^{*}$ such that $\Delta \psi^{*}$ belongs to $L^{p}(G)$ for all $p \in(1, \infty)$ and hence it can be represented by a function in $C^{1+\alpha}(G)$ for all $\alpha \in(0,1)$.

There exists a unique function $\Psi$ in $Y[9,15]$, such that

$$
P[\Psi]=\sup _{Y} P[u] \equiv \sup _{Y} \int_{G} u(x) d x,
$$

where $\Psi$ is referrred to as the completely plastic stress function. Let

$$
K^{+} \equiv\left\{u ; u \in H_{0}^{1}(G), 0 \leqq u \leqq \Psi \text { a.e. in } G\right\} .
$$


Then $K^{+}$is a closed convex subset of $H_{0}^{1}(G)$ and we have the following equivalent formulation for the loading problem [2], [15].

THEOREM 2.2. There is a unique $\psi$ in $K^{+}$such that

$$
I[\psi]=\inf _{K^{+}} I[u] \text { and } \psi=\psi^{*} \quad \text { in } \quad C^{1+\alpha}(G)
$$

This equivalent formulation provides certain conveniences in establishing the regularity results for $\psi$ as well as for setting up numerical schemes [2], [15]. Moreover, it gives a clear picture of how the domain $G$ is partitioned into elastic and plastic zones by the stress function $\psi$. Such information is not only essential for the existence of the warping function [1] but also for treating the unloading problem.

Since the minimizer $\psi$ belongs to $C^{1+\alpha}(G)$, the sets,

$$
\begin{aligned}
& E \equiv\{x ; x \in G,|\operatorname{grad} \psi(x)|<k\}, \\
& P \equiv\{x ; x \in G,|\operatorname{grad} \psi(x)|=k\},
\end{aligned}
$$

are well-defined. Moreover, we have the following equivalent characterization for $E$ and $P$ [15],

$$
E=\{x \text { in } G ; \psi(x)<\Psi(x)\}, \quad P=G \backslash E
$$

Denote by $\Gamma(\Psi)$ the set of all points in $G$ over which grad $\Psi$ is discontinuous. Then, under the restrictions (i) and (ii) on the domain $G$, $\Gamma(\Psi)$ consists of a finite number of smooth Jordan arcs [15a]. Moreover, we have [15c].

THEOREM 2.3. The open set $E$ is simply connected and contains the set $\Gamma(\Psi)$. The set $P$ consists of inward normal segments to $\partial G$.

Finally, we have the following results which ensures the existence of a unique warping function [1].

THEOREM 2.4. There exists a unique continuous function $\lambda(x)$ such that

$(2.9) \lambda(x) \geqq 0 \quad$ in $\quad G+\partial G, \quad \lambda(x)[k-|\operatorname{grad} \psi(x)|]=0 \quad$ on $\quad G+\partial G$, and such that

(2.10) $\int_{G}[(1+\lambda(x)) \nabla \psi \cdot \nabla u-2 \mu \theta u] d x=0 \quad$ for all $u$ in $H_{0}^{1}(G)$. 
3. Formulation of the unloading problem. Let $\psi$ be the minimizer of the loading problem (2.6) and $\lambda(x)$ be the corresponding Lagrange multiplier, as in Theorem 2.4. Let

$$
Z \equiv\left\{u \text { in } H_{0}^{1}(G) ; \int_{G} u(x) d x=0\right\} .
$$

Then $Z$ is a closed subspace of $H_{0}^{1}(G)$. The unloading problem for a elastically-plastically twisted bar with cross-section $G$ is to find a function $\zeta^{*}(x)$ in $Y \cap Z$ such that

$$
J\left[\zeta^{*}\right]=\inf _{Y \cap Z} J[u] \equiv \inf _{Y \cap Z} \int_{G}\left[|\nabla u|^{2}+2 \lambda \nabla \psi \cdot \nabla u\right] d x,
$$

where $Y$ is the closed convex set defined in (2.2).

Apparently, the above minimization principle is proposed here for the first time in accordance with the deformation theory in plasticity. Nevertheless, it is in agreement with other such principles which are based on the theory of Prandtl-Reuss [1].

It is also worthwhile to make a few observations before attacking the problem. Since the problem is formulated in terms of a single stress function $\zeta^{*}$, the conditions of equilibrium are automatically satisfied. Moreover, the restrictions on the admissible class of functions assure us two things. First, if an admissible function $u$ has mean value zero and vanishes on $\partial G$ then there is no applied terminal torque and the lateral surface of the bar is free from applied forces. Secondly, the modulus of the residual stress, $\left|\nabla \zeta^{*}\right|$, remains below the yield constant $k$. The minimization principle takes the place of the constitutive relations.

The term $\lambda(x) \nabla \psi(x) \cdot \nabla \zeta^{*}(x)$ in (3.2) represents the permanent deformation caused by the stress, $\nabla \Psi$, in the course of loading. If this term were omitted, then $\zeta^{*}$ would be identically equal to zero. In fact, if the multiplier $\lambda$ equals zero identically, which is possible if $\theta$ is sufficiently small, then $\zeta^{*}=0$ identically in $G$. Hence, in this case, there is no residual stress after unloading. This is just what one would expect from the plasticity theory. The problem so formulated can be solved explicitly if the domain $G$ is a circular disk.

Just as for the case of loading, it is desirable to have another formulation of the unloading problem. To do this, let

$$
K \equiv\left\{u \text { in } H_{0}^{1}(G) ;-\Psi \leqq u \leqq \Psi \text { a.e. in } G\right\},
$$

where $\Psi$ is the completely plastic stress function defined as the solution of problem (2.4). As is easily seen, $K$ is also a closed convex set in $H_{0}^{1}(G)$. Instead of finding $\zeta^{*}$, we consider the problem of finding a function $\zeta$ in $K \cap Z$ such that 


$$
J[\zeta]=\inf _{K \cap Z} J[u]
$$

Problem (3.4) has been so constructed that it is equivalent to problem (3.2) in the sense that these two problems have one and the same solution. However, this will not be proved until $\S 6$.

4. Existence and uniqueness of the solution. It turns out that these basic questions can be easily answered. Since the proof is elementary and since the reasoning used in the proof will also be useful later on, we present it in detail.

THEOREM 4.1. Both problems (3.2) and (3.4) have unique solutions.

Proof. Since the proof for the two problems is essentially the same, we carry it out for problem (3.4). Let $\left\{\zeta_{n}\right\}$ be a minimizing sequence of $J[u]$ over $K \cap Z$, i.e.,

$$
\lim _{n \rightarrow \infty} J\left[\zeta_{n}\right]=d=\inf J[u] \text { over } K \cap Z
$$

Clearly, $d$ is finite. Since $J[u]$ is defined on all of $H_{0}^{1}(G)$, simple computation leads to the identity

$$
J\left[\frac{1}{2}\left(\zeta_{m}+\zeta_{n}\right)\right]+\int_{G}\left|\nabla\left(\frac{1}{2}\left(\zeta_{m}-\zeta_{n}\right)\right)\right|^{2} d x=\frac{1}{2} J\left[\zeta_{m}\right]+\frac{1}{2} J\left[\zeta_{n}\right]
$$

Now, for every number $\epsilon>0$, we can, by virtue of (4.1), choose an integer $N$ so large that for all $m, n \geqq N$,

$$
J\left[\zeta_{n}\right] \leqq d+\epsilon / 2, \quad J\left[\zeta_{n}\right] \leqq d+\epsilon / 2
$$

On the other hand, $\left(\zeta_{m}+\zeta_{n}\right) / 2$ belongs to $K \cap Z$ for all values of $m$ and $n$. It follows that

$$
J\left[\left(\zeta_{m}+\zeta_{n}\right) / 2\right] \geqq d
$$

By replacing the 1st term on the left-hand side of (4.2) and the two terms on the right-hand side of (4.2) by their respective lower and upper bounds in (4.4) and (4.3), we obtain the estimates

$$
\frac{1}{4} \int_{G}\left|\operatorname{grad}\left(\zeta_{m}-\zeta_{n}\right)\right|^{2} d x \leqq \epsilon \text { for all } m \text { and } n \leqq N .
$$

Since $\epsilon$ is arbitrary, we conclude from (4.5) and Poincaré's inequality that the minimizing sequence $\left\{\zeta_{n}\right\}$ is actually a Cauchy sequence in $H_{0}^{1}(G)$. 
Denote by $\zeta$ the $\|\cdot\|_{1}$-limit of $\zeta_{n}$. Then, the Lebesgue dominated convergence theorem ensures that $J[\zeta]=d$. Since the function $\zeta$ so constructed belongs to $K \cap Z$, it is a minimizer of $J[u]$ over $K \cap Z$.

For the uniqueness, let $\zeta_{1}$ and $\zeta_{2}$ be two minimizers of $J[u]$ over $K \cap Z$. Then

$$
J\left[\zeta_{1}\right] \leqq J\left[\left(\zeta_{1}+\zeta_{2}\right) / 2\right], \quad J\left[\zeta_{2}\right] \leqq J\left[\left(\zeta_{1}+\zeta_{2}\right) / 2\right]
$$

By adding the corresponding sides of these two inequalities, we find

$$
\int_{G}\left|\operatorname{grad}\left(\zeta_{1}-\zeta_{2}\right)\right|^{2} d x \leqq 0
$$

By virtue of Poincaré's inequality, (4.6) holds if and only if $\zeta_{1}=\zeta_{2}$ in $H_{0}^{1}(G)$. The theorem is now established.

Having answered the basic question, we procceed to derive information about the solution. To this end, we first establish

5. The existence of a deformation multiplier. We wish to show that without destroying the minimization principle, a Lagrange multiplier can be introduced so as to eliminate one of the side constraints. Let $\omega$ be a parameter $\geqq 0$. Consider the one-parameter family of functionals,

$$
J_{\omega}[u] \equiv \int_{G}\left[|\nabla u|^{2}+2 \lambda \nabla \psi \cdot \nabla u-4 \mu \omega u\right] d x
$$

over the closed convex set $K$ in $H_{0}^{1}(G)$. By completely similar reasoning as used in the proof of Theorem 4.1, we have

THEOREM 5.1. For every $\omega \geqq 0$, there exists a unique function $\zeta_{\omega}$ in $K$ such that

$$
J_{\omega}\left[\zeta_{\omega}\right]=\inf _{K} J_{\omega}[u]
$$

Recall that the angle of twist per unit length at the end of loading is equal to $\theta$. We wish to show that if the loading is elastic-plastic, i.e., if $\theta$ is sufficiently large, then the bar remains partially twisted even after complete unloading. More precisely,

THEOREM 5.2. Suppose that the multiplier $\lambda(x) \not \equiv 0$. Then there is a

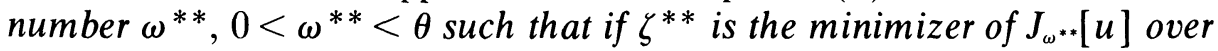
$K$, then $\zeta^{* *}=\zeta$ in $H_{0}^{1}(G)$, where $\zeta$ is the solution of problem (3.4). 
The multiplier $\omega$ represents the angle of twist per unit length of the completely unloaded bar relative to its natural (unstrained) state. If the permanent deformation occurred at at the end of the loading process, then the bar cannot recover its original shape after the removal of the applied torque. This physical reasoning indicates that $\omega^{* *}>0$. In particular, $\theta$ cannot decrease to zero in the process of unloading. Again, physical reasoning shows that the displacement of each material point must be reduced when the terminal couples are released. Accordingly, if our formulation of the problem does serve as a model of reality, then we must have $\omega^{* *}<\theta$. These are the physical reasonings leading to Theorem 5.2. For clarity, we split the proof into lemmas.

Lemma 5.3. Let $\zeta_{\omega}$ be the unique minimizer of problem (5.2). Then

$$
\omega \rightarrow f(\omega) \equiv \int_{G} \zeta_{\omega}(x) d x
$$

is continuous and nondecreasing on $[0, \infty)$.

Proof. Let $0<\omega_{1}<\omega_{2}$ be given numbers and let $\zeta_{1}$ and $\zeta_{2}$ be, respectively, the minimizers of $J_{\omega_{1}}[u]$ and $J_{\omega_{2}}[u]$ over the convex set $K$. Then for all $\epsilon$ in $[0,1]$, the function, $(1-\epsilon) \zeta_{1}+\epsilon \zeta_{2}$, lies in $K$. Hence, from the minimizing property of $\zeta_{1}$, we have

$$
d J_{\omega_{1}}\left[(1-\epsilon) \zeta_{1}+\epsilon \zeta_{2}\right] / d \epsilon \geqq 0 \quad \text { at } \quad \epsilon=0 .
$$

This means that

$$
\int_{G}\left[\left(\nabla \zeta_{1}+\lambda \nabla \psi\right) \cdot \nabla\left(\zeta_{2}-\zeta_{1}\right)-2 \mu \omega_{1}\left(\zeta_{2}-\zeta_{1}\right)\right] d x \geqq 0
$$

Similarly, we have

$$
\int_{G}\left[\left(\nabla \zeta_{2}+\lambda \nabla \psi\right) \cdot \nabla\left(\zeta_{1}-\zeta_{2}\right)-2 \mu \omega_{2}\left(\zeta_{1}-\zeta_{2}\right)\right] d x \geqq 0 .
$$

By adding these two inequalities together, we obtain

$$
2 \mu\left(\omega_{2}-\omega_{1}\right) \int_{G}\left(\zeta_{2}-\zeta_{1}\right) d x \geqq \int_{G}\left|\nabla\left(\zeta_{2}-\zeta_{1}\right)\right|^{2} d x \geqq 0
$$

which show that $f(\omega)$, defined in (5.3) is, indeed, a nondecreasing function of $\omega$.

To establish the continuity of $f$, we denote by $\operatorname{diam}(G)$ the diameter 
of $G$ and by $|G|$ the area of $G$. Similar notations will also be used later on for other measurable sets. Clearly,

$$
0 \leqq \Psi \leqq k \operatorname{diam}(G), \quad-\Psi \leqq \zeta_{1}, \zeta_{2} \leqq \Psi
$$

It follows immediately that

$$
\left|\zeta_{2}-\zeta_{1}\right| \leqq 2 k \operatorname{diam}(G)
$$

On the other hand, Poincaré's inequality states that there is a constant $C(G)$ depending only on $G$ such that

$$
C(G) \int_{G}\left|\nabla\left(\zeta_{2}-\zeta_{1}\right)\right|^{2} d x \geqq \int_{G}\left|\zeta_{2}-\zeta_{1}\right|^{2} d x
$$

A further application of the Schwartz inequality gives

$$
C(G)|G| \int_{G}\left|\nabla\left(\zeta_{2}-\zeta_{1}\right)\right|^{2} d x \geqq\left[\int_{G}\left(\zeta_{2}-\zeta_{1}\right) d x\right]^{2} .
$$

Now, it follows from (5.4)-(5.6) that

$$
4 k \mu C(G)\left(\omega_{2}-\omega_{1}\right)|G|^{2} \operatorname{diam}(G) \geqq\left[f\left(\omega_{2}\right)-f\left(\omega_{1}\right)\right]^{2}
$$

which ensures the continuity of $f$.

Actually, we can iterate the above procedure to prove the Lipschitz continuity of $f$, but we do not need it for later work.

REMARK 5.4. It is an open question whether $f(\omega)$ is a strictly increasing function of $\omega$ for all $\omega \geqq 0$. However, we shall establish the uniqueness of the deformation multiplier in $\S 8$.

Lemma 5.5. Let $f(\omega)$ be defined by equation (5.3). Then $f(0)<0$.

Proof. Denote by $\zeta_{0}$ the minimizer of $J_{\omega}[u]$ over $K$ with $\omega=0$. Since the minimizer $\psi$ of the loading problem belongs to $K^{+}$, the function $-\psi$ belongs to $K$. Consequently, for the minimizer $\zeta_{0}$, we have the variational inequality,

$$
\int_{G}\left[\nabla \zeta_{0} \cdot \nabla\left(\psi+\zeta_{0}\right)+\lambda \nabla \psi \cdot \nabla\left(\psi+\zeta_{0}\right)\right] d x \leqq 0
$$

It follows from this inequality and equation (2.10) that 


$$
\begin{aligned}
2 \mu \theta \int_{G} \zeta_{0}(x) d x & =\int_{G}\left[(1+\lambda) \nabla \psi \cdot \nabla \zeta_{0}\right] d x \\
& \leqq-\int_{G}\left[\left|\nabla \zeta_{0}\right|^{2}+\lambda|\nabla \psi|^{2}\right] d x<0
\end{aligned}
$$

This proves the lemma. We used the positivity of $\lambda(x)$ over a set of positive area in obtaining the last inequality. This means that the loading process does cause a "permanent set". Of course, this is what one would like to have.

Lemma 5.6. Let $f(\omega)$ be defined by formula (5.3). Then, $f(\omega)>0$ for all $\omega \geqq \theta$.

Proof. Since equation (2.10) holds for all $u$ in $H_{0}^{1}(G)$, the function $\psi$ minimizes the functional $J_{\theta}[u]$ over $H_{0}^{1}(G)$. If $u$ belongs to $H_{0}^{1}(G)$ with $u=\psi+w$, then

$$
J_{\theta}[u]-J_{\theta}[\psi]=\int_{G}(1+\lambda)|\nabla w|^{2} d x \geqq 0 .
$$

From this fact and the uniqueness of the minimizer for $J_{\theta}[u]$ over $H_{0}^{1}(G)$, we conclude that $\zeta_{\theta}=\psi$ in $H_{0}^{1}(G)$.

Recall that $\psi$ minimizes $I[u]$ over $K^{+}$. Hence, $\psi \geqq 0$ in $G$. Suppose that $\psi\left(x_{0}\right)=0$ at some point $x_{0}$ in $G$. Then, according to (2.7) and (2.8), the point $x_{0}$ lies in $E$ which is an open set contained in $G$. It follows from continuity that for some number $\epsilon>0,|\operatorname{grad} \psi|<k$ in the disk $D\left(x_{0}, \epsilon\right)$ centered at $x_{0} \cdot$ with radius $\epsilon$ such that $D\left(x_{0}, \epsilon\right) \subset E$. It is known [15c] that $\Delta \psi=-2 \mu \theta$ in $E$. We have, in particular,

$$
\Delta \psi=-2 \mu \theta \quad \text { in } \quad D\left(x_{0}, \epsilon\right), \quad \psi \geqq 0 \quad \text { on } \quad \partial D\left(x_{0}, \epsilon\right) .
$$

Now, $\psi\left(x_{0}\right)=0$ is an interior minimum of $\psi$ in the disk $D\left(x_{0}, \epsilon\right)$, and we have a contradiction with the strong maximum principle for the solutions of elliptic differential inequalities. Thus, we conclude that

$$
\psi(x)>0 \text { for all } x \text { in } G
$$

This inequality ensures that

$$
f(\theta) \equiv \int_{G} \zeta_{\theta}(x) d x=\int_{G} \psi(x) d x>0 .
$$

The fact that $f(\omega)>0$ for $\omega>\theta$ follows from Lemma 5.3. 
Proof of Theorem 5.2. We have shown that $f(\omega)$ defined in (5.3) is a continuous nondecreasing function of $\omega$ and that $f(0)<0$ and $f(\theta)>0$. Hence, there exists a number $\omega^{* *}, 0<\omega^{* *}<\theta$, such that if $\zeta^{* *}$ is the minimizer $J_{\omega^{* *}}[u]$ over $K$, then $f\left(\omega^{* *}\right)=0$.

To show that $\zeta^{* *}=\zeta$, we observe that $\zeta^{* *} \in K \cap Z$. Hence,

$$
\inf _{K} J_{\omega^{* *}}[u]=J_{\omega^{*}}\left[\zeta^{* *}\right]=\inf _{K \cap Z} J_{\omega^{* *}}[u]=\inf _{K \cap Z} J[u]
$$

Thus, both $\zeta$ and $\zeta^{* *}$ are the minimizers of the same functional $J[u]$ over the same closed convex set $K \cap Z$. It follows from the uniqueness of the minimizer that $\zeta^{* *}=\zeta$ in $H_{0}^{1}(G)$.

\section{Regularity of the minimizer and equivalence of} two formulations. Having established the existence of the deformation multiplier $\omega^{* *}$, we now regard $\zeta$ as the minimizer of $J_{\omega} \cdot[u]$ over $K$. To simplify notation, we write $\omega$ for $\omega^{* *}$ so that

$$
J_{\omega}[\zeta]=\inf _{K} \int_{G}\left\{|\nabla u|^{2}+2 \lambda \nabla \psi \cdot \nabla u-4 \mu \omega u\right\} d x .
$$

We wish to show that $\zeta$ belongs to $C^{1+\alpha}(G)$ for all $\alpha \in(0,1)$. As a consequence of the proof, we shall see that $|\nabla \zeta| \leqq k$ in $G$. The technique used in the proof is exactly the same as is given in [3]. However, the same device is applied to a different problem. Accordingly, we first describe the essential steps and then add some clarification.

THEOREM 6.1. If $\zeta$ is the minimizer of problem (6.1), then $\zeta \in$ $H_{0}^{2, p}(G)$ for all $p \in(1, \infty)$, and hence, it can be represented by a function in $C^{1+\alpha}(G)$ for all $\alpha \in(0,1)$.

Since $\zeta$ is the minimizer of $J_{\omega}[u]$ over $K$, we have the variational inequality,

$$
\int_{G}[(\nabla \zeta+\lambda \nabla \psi) \cdot \nabla(u-\zeta)-2 \mu \omega(u-\zeta)] d x \geqq 0
$$

for all $u$ in $K$. This inequality together with equation (2.10) implies that

$$
\int_{G}[\nabla(\zeta-\psi) \cdot \nabla(u-\zeta)+2 u(\theta-\omega)(u-\zeta)] d x \geqq 0
$$

for all $u$ in $K$. Inequality (6.3) is easier to handle, because it does not involve the multiplier $\lambda(x)$ which is not, in general, differentiable. To use 
variational inequality (6.3), we first construct a suitable elliptic differential operator. To do this, let

$$
\partial G_{r}=\{x \in \bar{G} \mid \operatorname{dist}(x, \partial G)<r\} .
$$

Since $\partial G$ is of class $C^{2}(\bar{G})$, there is $\left[3\right.$, p. 172] a function $\beta(x)$ in $C^{2}(\bar{G})$ such that

(6.4) $\beta(x)=k \operatorname{dist}(x, \partial G)$ on $\quad \partial G_{r}, \quad \beta(x) \geqq k \operatorname{dist}(x, \partial G)$ on $\quad G$, provided the positive number $r$ is small enough. Now, let

$$
\begin{aligned}
M & \equiv \max \left\{\sup \sum_{i, t} \partial^{2} \beta / \partial x_{\imath} \partial x_{j}, 0\right\}, \\
b_{\imath}(x) & \equiv M \partial \beta / \partial x_{\imath}, \quad \gamma>2 M / r, \\
A & \equiv \sum_{\imath} \partial^{2} / \partial x_{\imath} \partial x_{\imath}-\sum_{i} b_{\imath}(x) \partial / \partial x_{\imath}-\gamma .
\end{aligned}
$$

Having defined the operator $A$, we consider, for all $\epsilon>0$ and for all even integers $p>2$, the nonlinear Dirichlet problems,

$$
\epsilon A \zeta_{\epsilon}=\left(\zeta_{\epsilon}-\zeta\right)^{1 /(p-1)} \quad \text { in } \quad H_{0}^{1, p}(G) \cap H^{2, p}(G),
$$

for determining $\zeta_{\epsilon}$. Here $H^{k, p}(G)$ stands for the space of functions whose distribution derivatives of order $\leqq k$ are in $L^{p}(G)$. For the problems in (6.6), we have the following results.

1. For all $\epsilon>0$ and all even integers $p>2$, problem (6.6) has a solution $\zeta_{\epsilon}$ in $H_{0}^{1, p}(G) \cap H^{2, p}(G)$. This can be proved by considering the linear problems obtained by replacing $\zeta_{\epsilon}$ on the right-hand side in (6.6) by a given function in $H_{0}^{1, p}(G)$ and then apply Schauder's fixed-point theorem, $[8,15 \mathrm{c}]$.

2. If $\zeta_{\epsilon}$ is a solution of (6.6), then $-\beta<\zeta_{\epsilon}<\beta$ in $G$. This is a consequence of the weak maximum principle and its proof is similar to that given in [3, p. 174]. In fact, the operator $A$ was so constructed that $A \beta \leqq 0$ on $G$.

3. If $\zeta_{\epsilon}$ is a solution of (6.6) with $\gamma$ greater than a fixed number which depends only on $\beta$ and its first two derivatives, then $\left|\nabla \zeta_{\epsilon}\right| \leqq k$ in $G$. A detailed proof of this is given in [3, pp. 174-175].

4. If $\{\epsilon\}$ is a positive numerical sequence tending to zero and if $\left\{\zeta_{\epsilon}\right\}$ are the corresponding solutions of (6.6), then 


$$
\left\|\Delta \zeta_{\epsilon}\right\|_{0, p} \leqq \text { constant }
$$

which is independent of $\epsilon$. Here, $\|\cdot\|_{k, p}$ stands for the usual norm on $H^{k, p}(G)$.

Proof of assertion 4. We derive the last statement as a consequence of variational inequality (6.3). Since $\left|\nabla \zeta_{\epsilon}\right| \leqq k$ in $G$ for all small $\epsilon>0$, we may replace $u$ in $(6.3)$ by $\zeta_{\epsilon}$ to obtain

$$
-\int_{G}\left[\nabla\left(\zeta_{\epsilon}-\psi\right) \cdot \nabla\left(\zeta_{\epsilon}-\zeta\right)-2 \mu(\theta-\omega)\left(\zeta_{\epsilon}-\psi\right)\right] d x \leqq-\int_{G}\left|\nabla\left(\zeta_{\epsilon}-\zeta\right)\right|^{2} d x
$$

Applying the divergence theorem to the first integral on the left, we get

$$
\int_{G}\left(\Delta \zeta_{\epsilon}\right)\left(\zeta_{\epsilon}-\zeta\right) d x \leqq \int_{G}[2 \mu(\theta-\omega)+\Delta \psi]\left(\zeta_{\epsilon}-\zeta\right) d x
$$

Since the first equation in (6.6) can be written as

$$
\zeta_{\epsilon}-\zeta=\left(\epsilon A \zeta_{\epsilon}\right)^{p-1} \quad \text { in } \quad L^{q}(g), \quad q=p /(p-1)
$$

we can substitute this expression into $(6.8)$ for $\left(\zeta_{\epsilon}-\zeta\right)$ to obtain

$$
\int_{G}\left(\Delta \zeta_{\epsilon}\right)\left(A \zeta_{\epsilon}\right)^{p-1} d x \leqq \int_{G}[2 \mu(\theta-\omega)+\Delta \psi]\left(A \zeta_{\epsilon}\right)^{p-1} d x
$$

Applying the binomial theorem to expand $\left(A \zeta_{\epsilon}\right)^{p-1}$ into polynomials in $\Delta \zeta_{\epsilon}$, we can write (6.9) as follows:

$$
\int_{G}\left|\Delta \zeta_{\epsilon}\right|^{p} d x \leqq \int_{G}\left\{[2 \mu(\theta-\omega)+\Delta \psi] P_{p-1}\left(\Delta \zeta_{\epsilon}\right)+Q_{p-1}\left(\Delta \zeta_{\epsilon}\right)\right\} d x
$$

where $P_{p-1}\left(\Delta \zeta_{\epsilon}\right)$ and $Q_{p-1}\left(\Delta \zeta_{\epsilon}\right)$ are polynomials of degree $\leqq p-1$ in $\Delta \zeta_{\epsilon}$ with coefficients being monomials of degree $\leqq p$ in $\left(\gamma+\Sigma b_{i} \partial \zeta_{\epsilon} / \partial x_{i}\right)$. Since $\left|\nabla \zeta_{\epsilon}\right| \leqq k$ in $G$ and since $\beta \in C^{2}(\bar{G})$, it is clear that $\left|\gamma+\Sigma b_{i} \partial \zeta_{\epsilon} / \partial x_{i}\right|$ is bounded by a constant independent of $\epsilon$. Since $\psi \in H^{2, p}(G)$ for all $p \in(1, \infty)$, Hölder's inequality leads to the estimates,

$$
\left|\int_{G}(\Delta \psi)\left(\Delta \zeta_{\epsilon}\right)^{p-1} d x\right| \leqq\|\Delta \psi\|_{0, p}\left(\left\|\Delta \zeta_{\epsilon}\right\|_{0, p}\right)^{(p-1) / p}
$$

With these facts in mind, we now repeatedly apply Hölder's inequality to the right-hand side in (6.10) to obtain the desired estimate, 


$$
\left(\left\|\Delta \zeta_{\epsilon}\right\|_{0, p}\right)^{p} \leqq \text { const. } \sum_{j=0}^{p-1}\left(\left\|\Delta \zeta_{\epsilon}\right\|_{0, p}\right)^{\prime}
$$

where the constant is independent of $\epsilon$. This is possible, if and only if, inequality (6.7) holds.

Proof of Theorem 6.1. Let $\{\epsilon\}$ be a positive numerical sequence tending to zero and let $\left\{\zeta_{\epsilon}\right\}$ be the corresponding solutions of (6.6) with $p$ being an even integer $>2$. Then the estimates in (6.7) hold. Consequently, $\left\{\zeta_{\epsilon}\right\}$ is a bounded sequence in the reflexive Banach space $H_{0}^{1, p} \cap H^{2, p}(G)$. Hence, a subsequence of $\left\{\zeta_{\epsilon}\right\}$, which we also denote by $\left\{\zeta_{\epsilon}\right\}$, converges weakly on $H_{0}^{1, p} \cap H^{2, p}$ to a function $\hat{\zeta}$ in $H_{0}^{1, p} \cap H^{2, p}$. On the other hand, (6.6) and (6.7) imply that for $q=p /(p-1)$,

$$
\left(\left\|\zeta_{\epsilon}-\zeta\right\|_{0 . q}\right)^{q}=\left(\epsilon\left\|A \zeta_{\epsilon}\right\|_{0 . p}\right)^{p} \rightarrow 0 \quad \text { as } \quad \epsilon \rightarrow 0 .
$$

This shows that $\zeta$ is the strong limit of $\zeta_{\epsilon}$ on $L^{q}(G)$ as $\epsilon \rightarrow 0$. Since the injection map of $H_{0}^{1, p} \cap H^{2, p}(G)$ into $L^{q}(G)$ is continuous for $p>2$, we conclude that $\hat{\zeta}=\zeta$ in $H_{0}^{1, p} \cap H^{2, p}(G)$. By Sobolev's imbedding theorem, we also conclude that $\zeta \in C^{1+\alpha}(G)$ with $\alpha=1-2 / p$. Since $p$ is arbitrary, the proof is now complete.

As a consequence of the above proof, we have the following equivalence result which is essential for deriving the information given in $\S 11$ and $\S 12$.

TheOrem 6.2. Problems (3.2) and (3.4) are equivalent in the sense that a solution of one problem is a solution of the other.

Proof. Clearly, the set $Y$ defined in (2.2) is a subset of $K$ defined in (3.3). Hence,

$$
J_{\omega}[\zeta]=\inf _{K} J_{\omega}[u]=\inf _{K \cap Z} J[u] \leqq \inf _{Y \cap Z} J[u]=J\left[\zeta^{*}\right]
$$

Since the solutions to problems (3.2) and (3.3) are both unique, it suffices to show that $\zeta$ belongs to $Y$.

Let $\left\{\zeta_{\epsilon}\right\}$ be the sequence of solutions to problems (6.6). Then, for all $\epsilon>0,\left|\nabla \zeta_{\epsilon}\right| \leqq k$ everywhere in $G$. By Arzela's theorem, the subsequence of $\left\{\zeta_{\epsilon}\right\}$ can be so selected that $\zeta_{\epsilon}$ together with its first partial derivatives converges uniformly to $\zeta$ in $\bar{G}$ and that $|\nabla \zeta| \leqq k$. Thus, $\zeta$ belongs to $Y$ and the proof is complete.

Remark 6.3. Problem (3.2) has a nonlinear side constraint; while the side constraint for (3.4) is linear. Thus, it is not obvious that they are 
equivalent problems. It would be good, if this equivalence could be proved under weaker restrictions on $\partial G$, because it is the equivalent formulation in (3.4) that leads to the solution of elastic-plastic partition problem later in $\$ 11$ and the existence of the compatibility multiplier for the unloading problem. It is also worth to note that we have made use of equation (2.10) for the loading problem in deriving the variational inequality in (6.3).

\section{A natural admissibility condition and its conse-} quences. Having established the equivalence of the two formulations, we may now regard the residual stress function $\zeta$ either as the minimizer of $J[u]$ over $Y \cap Z$ or as the minimizer of $J_{\omega}[u]$ over $K$ according to our convenience. As a first consequence, we derive a natural admissibility condition for the unloading problem.

THEOREM 7.1. Let $\psi$ and $\zeta$ be the stress functions of the loading and the unloading problems, respectively. Then

$$
-\psi \leqq \zeta \leqq \psi \quad \text { everywhere in } \quad G \text {. }
$$

Proof. From equation (2.10), we see that $\psi$ minimizes the functional,

$$
I_{\lambda}[u] \equiv \int_{G}\left\{(1+\lambda)|\nabla u|^{2}-4 \mu \theta u\right\} d x
$$

over the whole space $H_{0}^{1}(G)$. In fact, for all $u$ in $H_{0}^{1}(G)$, we write $u=\psi+w$. Then

$$
\begin{aligned}
I_{\lambda}[u]-I_{\lambda}[\psi] & =\int_{G}\left\{2[(1+\lambda) \nabla \psi \cdot \nabla w-2 \mu \theta w]+|\nabla w|^{2}\right\} d x \\
& =\int_{G}(1+\lambda)|\nabla w|^{2} d x \geqq 0 .
\end{aligned}
$$

Hence, for all $u$ in $H_{0}^{1}(G)$, we have

$$
\int_{G}[(1+\lambda) \nabla \psi \cdot \nabla(u-\psi)-2 \mu \theta(u-\psi)] d x=0 .
$$

On the other hand, for the minimizer $\zeta$, we have for all $w$ in $K$,

$$
\int_{G}[(\nabla \zeta+\lambda \nabla \psi) \cdot \nabla(w-\zeta)-2 \mu \omega(w-\zeta)] d x \geqq 0 .
$$


Let $D^{+}$be the set of points in $G$ over which $\zeta>\psi$. Then, $D^{+}$is an open set properly contained in $G$. Now, defining

(7.4) $u(x)=\left\{\begin{array}{lll}\zeta(x) & \text { in } & D^{+}, \\ \psi(x) & \text { in } & G \backslash D^{+},\end{array} \quad w(x)=\left\{\begin{array}{lll}\psi(x) & \text { in } & D^{+}, \\ \zeta(x) & \text { in } & G \backslash D^{+},\end{array}\right.\right.$

and setting the test functions $u$ and $w$ in (7.2) and (7.3) equal to the ones defined in (7.4), we find

$$
\begin{aligned}
& \int_{D^{+}}[(1+\lambda) \nabla \psi \cdot \nabla(\zeta-\psi)-2 \mu \theta(\zeta-\psi)] d x=0, \\
& \int_{D^{+}}[(\nabla \zeta+\lambda \nabla \psi) \cdot \nabla(\psi-\zeta)-2 \mu \omega(\psi-\zeta)] d x \geqq 0 .
\end{aligned}
$$

By adding these two relations, we obtain

$$
\int_{D^{+}}\left[|\nabla(\zeta-\psi)|^{2}+2 \mu(\theta-\omega)(\zeta-\psi)\right] d x \leqq 0
$$

Since $\theta-\omega>0$ and $\zeta>\psi$ on $D^{+}$, this inequality holds only when $\left|D^{+}\right|=0$. This proves the second inequality in (7.1).

To establish the first inequality in (7.1), we let $D^{-} \equiv\{x \mid \zeta(x)<$ $-\psi(x)]$ and define

(7.5) $\quad u(x)=\left\{\begin{array}{lll}-\zeta(x) & \text { in } \quad D^{-}, \\ \psi(x) & \text { in } \quad G \backslash D^{-},\end{array} \quad w(x)=\left\{\begin{array}{lll}-\psi(x) & \text { in } & D^{-}, \\ \zeta(x) & \text { in } & G \backslash D^{-},\end{array}\right.\right.$

and then choose them as the test functions in (7.2) and (7.3), respectively. This gives

$$
\begin{aligned}
& \int_{D^{-}}[(1+\lambda) \nabla \psi \cdot \nabla(\zeta+\psi)-2 \mu \theta(\zeta+\psi)] d x=0 \\
& \int_{D^{-}}[(\nabla \zeta+\lambda \nabla \psi) \cdot \nabla(\zeta+\psi)-2 \mu \omega(\zeta+\psi)] d x \leqq 0
\end{aligned}
$$

By adding these variational conditions together, we get

(7.8) $\int_{D^{-}}\left[|\nabla(\zeta+\psi)|^{2}+2 \lambda \nabla \psi \cdot \nabla(\zeta+\psi)-2 \mu(\theta+\omega)(\zeta+\psi)\right] d x \leqq 0$

We wish to determine the sign of the integeral of $\lambda \nabla \psi \cdot \nabla(\zeta+\psi)$ 
over $D^{-}$. To this end, we note that $I[\psi]=\inf I[u]$ over the set $Y$. By Theorem 6.2, the unloading stress function $\zeta$ also belongs to $Y$. Consequently, if we choose $u(x)$ in (7.5) as the testing function for the minimizer $\psi$ of $I[u]$ over $Y$, then

$$
\int_{D^{-}}[\nabla \psi \cdot \nabla(\zeta+\psi)-2 \mu \theta(\zeta+\psi)] d x \leqq 0
$$

By combining this estimate with (7.6), we obtain

$$
\begin{aligned}
2 \mu \theta \int_{D^{-}}(\zeta+\psi) d x & =\int_{D^{-}}(1+\lambda) \nabla \psi \cdot \nabla(\zeta+\psi) d x \\
& \leqq 2 \mu \theta \int_{D^{-}}(\zeta+\psi) d x+\int_{D^{-}} \lambda \nabla \psi \cdot \nabla(\zeta+\psi) d x
\end{aligned}
$$

which shows that

$$
\int_{D^{-}} \lambda \nabla \psi \cdot \nabla(\zeta+\psi) d x \geqq 0
$$

Finally, it follows from this inequality and (7.8) that

$$
\int_{D}\left[|\nabla(\zeta+\psi)|^{2} d x-2 \mu(\theta+\omega)(\zeta+\psi)\right] d x \leqq 0
$$

Since $\zeta+\psi<0$ on $D^{-}$, the above inequality holds only when $\left|D^{-}\right|=0$. The theorem is now proved.

Having established Theorems 6.1 and 7.1, we derive useful information for the unloading problem. First, we recall the elastic region $E$ defined in (2.7) for the loading problem. We know that $E$ is a simply connected domain contained in $G$ and containing the ridge, $\Gamma(G)$, of $G$ [15c]. Under the restrictions (i) and (ii) as stated in $\$ 1$ on $G$, the boundary of $E, \partial E$, consists of a finite number of Jordan arcs. Moreover, each point on $\partial E$ is an end point of the unique inward normal segment to $\partial G$, which wholly lies in $P=G \backslash E$. Thus, $E$ is a rather nice Jordan domain for which the Dirichlet problem for the Poisson equation can always be solved by means of the Dirichlet principle [6]. It is this fact that enables us to derive what follows.

Consider the restrictions of both $\psi$ and $\zeta$ to the elastic region E. From Theorem 7.1 and the result for the loading problem, we conclude that

$$
-\Psi(x)<-\psi(x) \leqq \zeta(x) \leqq \psi(x)<\Psi(x)
$$


everywhere in $E$. Since $\lambda(x)=0$ identically in $E$, the restriction of $\zeta$ to $E$ minimizes the functional,

$$
\int_{E}\left\{|\nabla u|^{2}-4 \mu \omega u\right\} d x
$$

over the closed convex set

$$
K_{E} \equiv\left\{u \mid u \in H^{1}(E), u=\zeta \text { a.e. on } \partial E,-\Psi \leqq u \leqq \Psi\right\}
$$

Because of the two strict inequalities in (7.9), it follows from standard analysis $[5,6,12,15]$ that .

THEOREM 7.2. If $E$ is the elastic region of $G$ at the end of loading and if $\zeta$ is the residual stress function after complete unloading, then $\zeta$ is analytic in $E$ and $\Delta \zeta=-2 \mu \omega$ in $E$.

Remark 7.3. Since $\zeta$ satisfies the Poisson equation in $E$, the compatibility conditions for the existence of the warping function over $E$ are satisfied $[\mathbf{1 1}, \mathbf{1 5}]$. Moreover, the residual stress and the residual strain relations in $E$ are still given by Hooke's law. As we shall see later on, $|\operatorname{grad} \zeta|<k$ everywhere in $E$. Consequently, the material in $E$ remains elastic after complete unloading. This is, of course, what one would expect.

\section{The uniqueness of the deformation multiplier. In} $\S 5$, we established the existence of a deformation multiplier $\omega, 0<\omega<\theta$, under the assumption that $\lambda(x)>0$ over a set of positive area. The multiplier $\omega$ represents the angle of twist of the unloaded bar with reference to the natural state. We left the uniqueness question open. Now, it is easy to prove it by negation.

Suppose that there are two deformation multipliers, $\omega_{1}, \omega_{2}$ such that $0<\omega_{1}<\omega_{2}<\theta$. Let $\zeta_{1}$ and $\zeta_{2}$ be, respectively, the minimizers of problem (6.1) with $\omega=\omega_{1}$ and $\omega_{2}$. Then, all the preceding analyses hold for both $\zeta_{1}$ and $\zeta_{2}$. By Lemma 5.3, $\zeta_{2} \geqq \zeta_{1}$ in $G$. This, together with Theorem 7.1 and the results on loading process, implies that

$$
-\Psi<-\psi \leqq \zeta_{1} \leqq \zeta_{2} \leqq \psi<\Psi \quad \text { in } \quad E
$$

In particular, these inequalities hold on $\partial E$. By Theorem 7.2, the function $\zeta_{2}-\zeta_{1}$ satisfies the Poisson equation

$$
\Delta\left(\zeta_{2}-\zeta_{1}\right)=-2 \mu\left(\omega_{2}-\omega_{1}\right) \text { in } E \text {. }
$$


By hypothesis, $\omega_{2}-\omega_{2}>0$. It follows from the strong maximum principle for the solution of elliptic differential inequalities that $\zeta_{2}>\zeta_{1}$ everywhere in $E$. Since $|E|>0$,

$$
\int_{G}\left(\zeta_{2}-\zeta_{1}\right) d x=\int_{P}\left(\zeta_{2}-\zeta_{1}\right) d x+\int_{E}\left(\zeta_{2}-\zeta_{1}\right) d x>0 .
$$

This contradicts the fact that the integrals of both $\zeta_{1}$ and $\zeta_{2}$ over the domain $G$ are both equal to zero. Hence, the assumption, $\omega_{1} \neq \omega_{2}$, is wrong, and hence, the uniqueness of the deformation multiplier is proved.

Although, the fact, $|E|>0$, is decisive for the uniqueness of the deformation multiplier $\omega$, it was proved in [15c] that the elastic core $E$ always exists provided $G$ is simply connected.

9. A minimization principle for stress decrement. Because of the presence of permanent deformation, we cannot expect the complete release of the stress after complete unloading. However, on the ground of physical reasoning, it is expected that the unloading process would reduce the modulus of the stress. Accordingly, we formulate a minimization principle for direct determination of $\zeta-\psi$. Also, this principle will be used later in $\$ 11$ in solving the elastic-plastic partition problem.

To formulate the principle, we write, for all $u$ in $K$,

$$
u=\psi+w
$$

Then, $w$ also lies in $H_{0}^{1}(G)$. In fact, $w$ lies in the translate of $K$ by the minimizer $\psi$. Moreover,

$$
\begin{aligned}
J_{\omega}[u]= & \int_{G}\left[|\nabla(\psi+w)|^{2}+2 \lambda \nabla \psi \cdot \nabla(\psi+w)-4 \mu \omega(\psi+w)\right] d x \\
= & \int_{G}\left[|\nabla w|^{2}+4 \mu(\theta-\omega) w\right] d x \\
& +\int_{G} 2[(1+\lambda) \nabla \psi \cdot \nabla w-2 \mu \theta w] d x \\
& +\int_{G}\left[(2 \lambda+1)|\nabla \psi|^{2}-4 \mu \omega \psi\right] d x .
\end{aligned}
$$

Since the value of the second integral is zero and that of the third integral is a constant independent of the function $w$, we have established

THEOREM 9.1. Let $\psi$ and $\zeta$ be, respectively, the solutions of the 
loading and the unloading problems and let $\chi=\zeta-\psi$. Then, $\chi$ minimizes the functional,

$$
I_{\theta-\omega}[u] \equiv \int_{G}\left[|\nabla w|^{2}+4 \mu(\theta-\omega) w\right] d x,
$$

over the closed convex subset,

$$
K_{\psi} \equiv\left\{w \mid w \in H_{0}^{1}(G),-(\Psi+\psi) \leqq w \leqq \Psi-\psi \text { a.e. }\right\}
$$

of $H_{0}^{1}(G)$.

10. The existence of elastic core. For the loading problem in (2.6), the existence of an elastic core $E$ is always assured [15c]. We wish to show that this elastic region $E$ remains elastic after the removal of the terminal couples.

THEOREM 10.1. Let $E$ be the elastic region at the end of the loading process and let $\zeta$ be the minimizer of the unloading problem, then everywhere in $E$

$$
|\nabla \zeta|<k, \quad-\psi<\zeta<\psi
$$

Proof. Theorem 6.2 ensures that $|\nabla \zeta| \leqq k$ everywhere in $\bar{G}$. In particular, $|\nabla \zeta| \leqq k$ everywhere on $\partial E$. By Theorem 7.2, $\zeta$ is analytic and satisfies the equation, $\Delta \zeta=-2 \mu \omega$, in $E$. Hence, we may appeal to computation to verify that

\section{$\Delta|\nabla \zeta|^{2}>0$ everywhere in $E$.}

Consequently, the strong maximum principle for the solutions of elliptic differential inequalities [12] ensures that $|\nabla \zeta|$ is strictly less than $k$ in $E$.

Since $\psi$ is analytic and satisfies the equation, $\Delta \psi=-2 \mu \theta$, in $E$ with $\theta>\omega$ and since $-\psi \leqq \zeta \leqq \psi$ in $G$, the functions $\psi-\zeta$ and $\psi+\zeta$ satisfy the following equations:

$$
\begin{aligned}
& \Delta(\psi-\zeta)=-2 \mu(\theta-\omega)<0 \quad \text { in } \quad E, \quad \psi-\zeta \geqq 0 \text { on } \partial E \text {, } \\
& \Delta(\psi+\zeta)=-2 \mu(\theta+\omega)<0 \quad \text { in } \quad E, \quad \psi+\zeta \geqq 0 \text { on } \partial E \text {. }
\end{aligned}
$$

Again, as the solutions of problems (10.2) and (10.3), respectively, both $\psi-\zeta$ and $\psi+\zeta$ must, by virtue of the strong maximum principle, be strictly positive in $E$. The inequalities in (10.1) are now proved.

REMARK 10.2. After complete unloading, the simply connected set 
$E$ remains elastic in the sense that (i) the modulus of the residual stress is strictly less than the yield constant and (ii) the relations between the residual stress and the strain referred to the natural state are governed by Hooke's law.

11. Elastic-plastic partition of the unloaded bar. With the regularity theorem on hand, we may partition the cross section of the unloaded bar into the elastic zones and plastic zones according to the stress and strain relations. To this end, we first establish the following theorem, which is a sharpened version of the second inequality in Theorem 7.1.

THEOREM 11.1. Let $\psi$ and $\zeta$ be the loading and the unloading stress functions, respectively. Then $\zeta<\psi$ everywhere in $G$.

Proof. By Theorem 7.1. $\chi=\zeta-\psi \leqq 0$ everywhere in G. Accordingly, we need only to show that $\chi<0$ in $G$. To do this we assume the contrary and then derive a contradiction.

Let $x_{0}$ be a point in $G$ such that

$$
\chi\left(x_{0}\right)=0 \text { and }(\Psi+\psi)\left(x_{0}\right)=C>0 .
$$

Let $D\left(x_{0}, r_{0}\right)$ be a disk centered at $x_{0}$ with radius $r_{0}$ so small that $D\left(x_{0}, r_{0}\right) \subset G$. Let $(r, \phi)$ be plane polar coordinates with origin at $x_{0}$. Consider the Dirichlet problem:

$$
\Delta w=2 \mu(\theta-\omega) \quad \text { in } \quad D\left(x_{0}, r_{0}\right), \quad w=\chi \quad \text { on } \quad \partial D\left(x_{0}, r_{0}\right) .
$$

Then $w$ is explicitly given by the formula, [7],

$$
w(r, \phi)=\frac{1}{2} \mu(\theta-\omega)\left(r^{2}-r_{0}^{2}\right)+\frac{1}{2 \pi} \int_{-\pi}^{\pi} \frac{\left(r_{0}^{2}-r^{2}\right) \chi\left(r_{0}, \sigma\right) d \sigma}{r_{0}^{2}-2 r \cos (\phi-\sigma)+r^{2}} .
$$

Since $\chi\left(r_{0}, \sigma\right) \leqq 0$ for $|\sigma| \leqq \pi$, the formula shows that $w(r, \phi)<0$ in $D\left(x_{0}, r_{0}\right)$. In particular, $w(0, \phi)<0$.

We proceed to derive a lower bound for $w$ so as to ensure that the function $w$ so constructed will lie in the admissible class of the restriction of the minimizer $\chi$ on $D\left(x_{0}, r_{0}\right)$. Clearly, $0 \geqq \chi\left(r_{0}, \sigma\right) \geqq-k r_{0}$. Hence,

$$
\begin{aligned}
0 \geqq w(r, \phi) & \geqq \frac{1}{2} \mu(\theta-\omega)\left(r^{2}-r_{0}^{2}\right)-\frac{k r_{0}}{2 \pi} \int_{-\pi}^{\pi} \frac{\left(r_{0}^{2}-r^{2}\right) d \sigma}{r_{0}^{2}-2 r \cos (\phi-\sigma)+r^{2}} \\
& =\frac{1}{2} \mu(\theta-\omega)\left(r^{2}-r_{0}^{2}\right)-k r_{0} \\
& \geqq-\frac{1}{2} \mu(\theta-\omega) r_{0}^{2}-k r_{0} .
\end{aligned}
$$


Now, we choose $r_{0}$ so small that

$$
w(r, \phi) \geqq-[\Psi(r, \phi)+\psi(r, \phi)] \quad \text { everywhere in } D\left(x_{0}, r_{0}\right) .
$$

This is so, if

$$
C>\frac{1}{2} \mu(\theta-\omega) r_{0}^{2}+k r_{0}
$$

Since $C$ is a fixed constant for fixed choice of $x_{0}$ in $G,(11.4)$ holds if $r_{0}$ is less than a fixed positive number.

Finally, if $w$ is the solution of problem (11.2) with $r_{0}$ satisfying inequality (11.4), then the Dirichlet principle states that

$$
\int_{D\left(x_{0}, r_{0}\right)}\left[|\nabla w|^{2}+4 \mu(\theta-\omega) w\right] d x \leqq \int_{D\left(x_{0}, r_{0}\right)}\left[|\nabla \chi|^{2}+4 \mu(\theta-\omega) \chi\right] d x .
$$

Consequently, the uniqueness of the minimizer demands that $w=\chi$ everywhere in $D\left(x_{0}, r_{0}\right)$. Thus, we reach the contradiction that $0=$ $\chi(0, \phi)<0$. The theorem is thereby proved. follows:

Having established the above theorem, we now partition $G$ as

$$
\begin{aligned}
& E_{u} \equiv\{x \in G ;-\Psi(x)<\zeta(x)<\Psi(x)\}, \\
& P_{u} \equiv\{x \in G ; \zeta(x)=-\Psi(x)\},
\end{aligned}
$$

and call them the elastic and the plastic zones of $G$. By Theorem 7.1, we know that $-\Psi \leqq \zeta \leqq \Psi$ everywhere in $G$. Hence

$$
E_{u} \cap P_{u}=0 \quad \text { and } \quad E_{u} \cup P_{u}=G .
$$

Thus, $E_{u}$ and $P_{u}$ does constitute a partition for $G$. Moreover, $E_{u} \supset E$. This means that the elastic region $E$ at the end of loading remains elastic after complete unloading. This is what has been shown before. Although, for some domain $G$ such as circular disk, $P_{u}$ may be empty. However, if $G$ possesses a re-entrant corner, then $P_{u}$ is nonempty.

Finally, we mention that the sets,

$$
E_{u}^{*} \equiv\{x \in G ;|\operatorname{grad} \zeta(x)|<k\}, \quad P_{u}^{*} \equiv G \backslash E_{u}^{*},
$$

also constitute a partition of $G$. It is easy to see that $P_{u} \subset P_{u}^{*}$ and hence $E_{u}^{*} \subset E_{u}$. In the case of loading, the above two types of partition turns out to be identical. It is not known, however, whether this remains true for the unloading problem. 
We proceed to establish the next theorem which shows the relative position of $E_{u}$ in $G$ as well as its extent in $G$. Moreover, the truth of the next theorem is decisive for the existence of a unique compatibility multiplier and hence for the existence of a warping function which is unique up to a constant.

THEOREM 11.2. The elastic region $E_{u}$ is a simply connected domain containing the ridge $\Gamma(G)$ of $G$; the plastic zone $P_{u}$ consists of the inward normal segments of $\partial G$, i.e., the intersection of $P_{u}$ with any inward normal to $\partial G$ is either empty or is a single segment with one end point on $\partial G$.

Proof. Suppose that $x_{0} \in P_{u}$ and that $\zeta\left(x_{0}\right)=-\Psi\left(x_{0}\right)$. Since $P_{u}=$ $G \backslash E_{u}$ and since $E_{u} \supset E \supset \Gamma(G)$, there exists a unique point $y_{0} \in \partial G$ such that the segment $x_{0} y_{0}$ lies in $G$ and is perpendicular to $\partial G$. We assert that segment $x_{0} y_{0}$ is contained in $P_{u}$. In fact, if this were not the case, then we could apply the mean-value theorem in differential calculus to derive a contradiction to the fact established in Theorem 6.2 that $|\operatorname{grad} \zeta| \leqq k$ in $G \cup \partial G$. This proves the second part of the theorem.

For the simple connectivity of $E_{u}$, we recall that $\Gamma(G)$ is a simply connected set contained in $G[\mathbf{1}, \mathbf{1 5} \mathrm{c}]$. Moreover, for every point $\gamma \in \Gamma(G)$, there exists at least one point, in general two points, $s$ on $\partial G$ such that $\operatorname{dist}(\gamma, s)=\operatorname{dist}(\gamma, \partial G)$ and that the segment $\gamma s$ lies in $G$. From the intersection property of $P_{u}$, we see that any two points in $E_{u}$ can be connected by a portion of $\Gamma(G)$ and the inward normal segments to $\partial G$ through the two points in $E_{u}$. The proof is now complete.

12. The existence of residual-displacement field. We have seen in Theorem 7.2 that grad $\zeta$ satisfies the Beltrami-Michell compatibility conditions, $\Delta \zeta=-2 \mu \omega$, in $E$, [15g]. Consequently, the existence of a warping function unique up to an additive constant is assured in $E$.

Consider now the "annular region", $E_{u} \backslash E$. Since $-\Psi<\zeta<\Psi$ in $E_{u} \backslash E$, the minimizer of problem (6.1) is free from any side constraint in $E_{u} \backslash E$. Hence, for any function $u$ in $H_{0}^{1}\left(E_{u} \backslash E\right)$, the variational equality,

$$
\int_{E_{u} \backslash E}\{(\nabla \zeta+\lambda \nabla \psi) \cdot \nabla u-2 \mu \omega u\} d x=0
$$

holds. Since this is precisely the compatibility condition for the stress field, $\nabla \zeta+\lambda \nabla \psi$, in $E_{u} \backslash E$, see e.g. [11], to have a corresponding displacement in accordance with Hooke's law, the existence of a unique warping function in $E_{u} \backslash E$ is also assured. Because of the presence of the terms $\lambda \nabla \psi$, which represents "plastic strain", Hooke's law holds in $E_{u} \backslash E$ only when the residual strain is referred to the permanently deformed state. 
But this is just the special feature of the plasticity theory. I also emphasized that it is in this sense that $E_{u} \backslash E$ is said to be elastic.

Finally, we consider the plastic zone $P_{u}$. As was already emphasized, Theorem 10.2 ensures, by the same analysis given in [1], the existence of a unique compatibility multiplier $\lambda_{u}(x)$ such that

$$
\begin{aligned}
& \lambda_{u}(x)=0 \text { in } E_{u}, \quad \lambda_{u}(x)>0 \text { in } P_{u}, \\
& \int_{G}\left\{\left[\left(1+\lambda_{u}\right) \nabla \zeta+\lambda \nabla \psi\right] \cdot \nabla u-2 \mu \omega u\right\} d x=0
\end{aligned}
$$

for all $u$ in $H_{0}^{1}(G)$. It follows that there exists a unique warping function in $P_{u}$. Needless to say, the warping function matches continuously along $\partial\left(E_{u} \mid E\right)$.

\section{REFERENCES}

1. H. Brezis, Multiplicateur de Lagrange en torsion "elasto-plastique", Arch. Rat. Mech. Anal., 49 (1972), 32-40; Problémes unilatéraux, J. Math. Pures and Appl., 51 (1972), 1-68.

2. H. Brezis and M. Sibony, Equivalence de deux inéquations variationelles et applications, Arch. Rat. Mech. Anal., 41 (1971), 254-265.

3. H. Brezis and G. Stampacchia, Sur la régularité de la solution d'inéquations elliptiques, Bull. Soc. Math. France, 96 (1968), 153-180.

4. G. Duvaut and J. L. Lions, Les inéquations en mécanique et en physique, Dunod, Paris, 1972, Chapt. 5.

5. G. Fichera, Existence theorems in elasticity, Handbuch der Physik, VI a/2, Springer-Verlag, New York, 1972, pp. 347-389.

6. P. R. Garabedian, Partial differential equations, John Wiley and Sons, New York, 1964.

7. P. R. Garabedian, H. Lewy and M. Schiffer, Axially symmetric cavitational flow, Ann. Math., 56 (1952), 560-602.

8. H. Lewy and G. Stampacchia, On the regularity of the solution of a variational inequality, Comm. Pur. Appl. Math., 22 (1969), 153-188.

9. H. Lanchon, Torsion elastoplastique d'un arbre cylindrique de section simplement ou multiplement connexe, J. Mech., 13 (1974), 267-320.

10. H. Lanchon and G. Duvaut, Sur la solution du problème de la torsion elasto-plastique d'une barre cylindrique de section quelconque, C. R. Acad. Sce. Paris, Ser A., 24 (1967), 502-503.

11. W. Prager and P. G. Hodge Jr., Theory of perfectly plastic solids, John Wiley and Sons, New York, 1951.

12. M. H. Protter and H. F. Weinberger, Maximum Principles in Differential Equations, PrenticeHall, Englewood, NJ, 1967.

13. G. Stampacchia, Regularity of solutions of some variational inequalities, Non-linear functional analysis, (Proc. Symp. Pure Math.) Vol. 18, Part I., (1970), 271-281.

14. T. Y. Thomas, Plastic Flow and Fracture in Solids, Academic Press, New York, 1961.

15. T. W. Ting, (a) The ridge of a Jordan domain and completely plastic torsion, J. Math. Mech., 15 (1966), 15-46; (b) Elastic-plastic torsion of a square bar, Trans. Amer. Math. Soc., 123 (1966), 369-401; (c) Elastic-plastic torsion of simply connected cylindrical bars, Indiana U. Math. J., 20 (1971), 1047-1076; (d) Torsional rigidities in the elastic-plastic torsion of simply connected cylindrical bars, Pacific J. Math., 46 (1973), 257-267; (e) Torsional rigidities of bars under fully plastic torsion, SIAM J. Appl. Math., 25 (1973), 54-68. (f) St. Venant's compatibility conditions, Tensor, N. S., 28 (1974), 5-12; 
(g) St. Venant's compatibility conditions and basic problems in elasticity (to appear in Rocky Mountain J. Math).

16. C. Truesdell and W. Noll, The non-linear field theories of mechanics, Encyclopedia of physics, Vol. III-3, Springer-Verlag, New York, 1965.

17. B. De St. Venant, Mémoire sur la torsion des prisms, Mem. div. Sav. Acad. Sci., 14 (1856), 233-560.

18. H. F. Weinberger, Upper and lower bounds for torsional rigidity, J. Math. Phys., 32 (1953), 54-62.

Received July 9, 1975 and in revised form April 6, 1976. Work supported in part by NSF Grant no. MPS 75-07118.

University of ILlinois at Champaign-Urbana 


\section{PACIFIC JOURNAL OF MATHEMATICS}

\section{EDITORS}

RICHARD ARENS (Managing Editor)

University of California

Los Angeles, California 90024

\author{
R. A. Beaumont \\ University of Washington \\ Seattle, Washington 98105
}

\section{J. DugunduI}

Department of Mathematics University of Southern California Los Angeles, California 90007

D. Gilbarg and J. Milgram

Stanford University

Stanford, California 94305

\section{ASSOCIATE EDITORS}

E. F. BECKENBACH
B. H. NeumanN

F. Wolp
K. YoshidA

\section{SUPPORTING INSTITUTIONS}

UNIVERSITY OF BRITISH COLUMBIA

CALIFORNIA INSTITUTE OF TECHNOLOGY

UNIVERSITY OF CALIFORNIA

MONTANA STATE UNIVERSITY

UNIVERSITY OF NEVADA

NEW MEXICO STATE UNIVERSITY

OREGON STATE UNIVERSITY

UNIVERSITY OF OREGON

OSAKA UNIVERSITY

\author{
UNIVERSITY OF SOUTHERN CALIFORNIA \\ STANFORD UNIVERSITY \\ UNIVERSITY OF HAWAII \\ UNIVERSITY OF TOKYO \\ UNIVERSITY OF UTAH \\ WASHINGTON STATE UNIVERSITY \\ UNIVERSITY OF WASHINGTON \\ AMERICAN MATHEMATICAL SOCIETY
}

The Supporting Institutions listed above contribute to the cost of publication of this Journal, but they are not owners or publishers and have no responsibility for its contents or policies.

Mathematical papers intended for publication in the Pacific Journal of Mathematics should be in typed form or offset-reproduced (not dittoed). double spaced with large margins. Underline Greek letters in red, German in green, and script in blue. The first paragraph or two must be capable of being used separately as a synopsis of the entire paper. Items of the biblography should not be cited there unless absolutely necessary, in which case they must be identified by author and Journal, rather than by item number. Manuscripts, in duplicate, may be sent to any one of the four editors. Please classify according to the scheme of Math. Reviews, Index to Vol. 39. All other communications should be addressed to the managing editor, or Elaine Barth, University of California, Los Angeles, California, 90024.

100 reprints are provided free for each article, only if page charges have been substantially paid. Additional copies may be obtained at cost in multiples of 50 .

The Pacific Journal of Mathematics is issued monthly as of January 1966. Regular subscription rate: $\$ 72.00$ a year (6 Vols., 12 issues). Special rate: $\$ 36.00$ a year to individual members of supporting institutions.

Subscriptions, orders for back numbers, and changes of address should be sent to Pacific Journal of Mathematics, 103 Highland Boulevard, Berkeley, California, 94708.

PUBLISHED BY PACIFIC JOURNAL OF MATHEMATICS, A NON-PROFIT CORPORATION Printed at Jerusalem Academic Press, POB 2390, Jerusalem, Israel.

Copyright (C) 1976 Pacific Journal of Mathematics All Rights Reserved 


\section{Pacific Journal of Mathematics}

\section{Vol. 64, No. $2 \quad$ June, 1976}

Richard Fairbanks Arnold and A. P. Morse, Plus and times............. 297

Edwin Ogilvie Buchman and F. A. Valentine, External visibility ......... 333

R. A. Czerwinski, Bonded quadratic division algebras.............. 341

William Richard Emerson, Averaging strongly subadditive set functions in unimodular amenable groups. II .........................

Lynn Harry Erbe, Existence of oscillatory solutions and asymptotic behavior for a class of third order linear differential equations ............. 369

Kenneth R. Goodearl, Power-cancellation of groups and modules........ 387

J. C. Hankins and Roy Martin Rakestraw, The extremal structure of locally compact convex sets ...................................

Burrell Washington Helton, The solution of a Stieltjes-Volterra integral

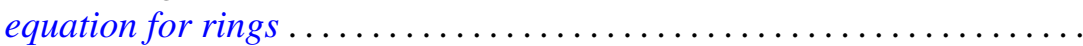

Frank Kwang-Ming Hwang and Shen Lin, Construction of 2-balanced

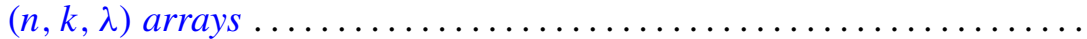

Wei-Eihn Kuan, Some results on normality of a graded ring ... 455

Dieter Landers and Lothar Rogge, Relations between convergence of series and convergence of sequences ......................... 465

Lawrence Louis Larmore and Robert David Rigdon, Enumerating immersions and embeddings of projective spaces ................

Douglas C. McMahon, On the role of an abelian phase group in relativized problems in topological dynamics..................

Robert Wilmer Miller, Finitely generated projective modules and TTF classes...

Yashaswini Deval Mittal, A class of isotropic covariance functions ...

Anthony G. Mucci, Another martingale convergence theorem ...

Joan Kathryn Plastiras, Quasitriangular operator algebras ...

John Robert Quine, Jr., The geometry of $p\left(S^{1}\right) \ldots \ldots \ldots$. 